\title{
Electromagnetic Impurity-Immunity Induced by Parity-Time Symmetry
}

\author{
Jie Luo, ${ }^{1,2}$ Jensen $\mathrm{Li}^{3,4, \dagger}$ and Yun Lai ${ }^{1,2, *}$ \\ ${ }^{1}$ National Laboratory of Solid State Microstructures, School of Physics, \\ and Collaborative Innovation Center of Advanced Microstructures, \\ Nanjing University, Nanjing 210093, China \\ ${ }^{2}$ College of Physics, Optoelectronics and Energy \& Collaborative Innovation Center of Suzhou Nano \\ Science and Technology, Soochow University, Suzhou 215006, China \\ ${ }^{3}$ Department of Physics, The Hong Kong University of Science and Technology, \\ Clear Water Bay, Hong Kong, China \\ ${ }^{4}$ William Mong Institute of Nano Science and Technology, The Hong Kong University of Science \\ and Technology, Clear Water Bay, Hong Kong, China
}

(Received 29 September 2017; revised manuscript received 8 June 2018; published 3 August 2018)

\begin{abstract}
Impurities usually play an important role in modifying the bulk properties of electronic or electromagnetic materials. In this work, we demonstrate a way to break this discipline and realize the extraordinary physical property of impurity-immunity, which leads to perfect transmission irrespective of embedded impurities of almost any material and shape. This extraordinary property comes from the exceptional points of a pair of parity-time $(P T)$-symmetric metasurfaces sandwiching a slab of epsilonnear-zero medium. By systematically investigating the $P T$-symmetric metasurfaces sandwiching a slab of dielectrics or metamaterials, we obtain the two complementary solutions of exceptional points corresponding to perfect transmission. Interestingly, when the permittivity of the slab approaches zero, the two solutions of exceptional points coalesce into one exceptional point. At such a critical point, we find that the original "doping" effect of impurities in epsilon-near-zero media, proposed by Nader Engheta's group very recently [I. Liberal et al., Science 355, 1058 (2017)], is significantly suppressed. Our work shows that exceptional points can be used to eliminate scattering of impurities in a bulk medium.
\end{abstract}

DOI: 10.1103/PhysRevX.8.031035

Impurities usually play an important role in modifying the bulk properties of electronic or electromagnetic materials. Doping, i.e., intentionally introducing impurities into a pure material, is of vital importance in the development of the semiconductor industry. Similar phenomena have been observed in the field of electromagnetic materials. Effective medium theories [1-3] have been developed to predict the modified bulk properties of electromagnetic media embedded with impurities in difference scenarios. Very recently, it has been observed that the bulk properties, as well as transmission behaviors of epsilon-near-zero (ENZ) media [4-22], are very sensitive to embedded impurities. Based on this effect, a "doping" method has been proposed to control the impedance of ENZ media [4].

\footnotetext{
* Corresponding author. laiyun@nju.edu.cn

Corresponding author. jensenli@ust.hk

Published by the American Physical Society under the terms of the Creative Commons Attribution 4.0 International license. Further distribution of this work must maintain attribution to the author(s) and the published article's title, journal citation, and DOI.
}

Subject Areas: Metamaterials

Even though sensitivity on impurities is general and useful in many scenarios, the pursuit of impurity-immunity or antidoping, i.e., independence of impurities including nonlinear impurities [23,24], has always been a hot research topic in physics. This is mainly because impurity-immunity could lead to robust high transmission of energy against small disturbances, which is of vital importance in many applications. Recent investigation of topological insulators has provided an approach to realize impurity-immunity for surface waves, which are robust against impurities and defects on the surface. Tremendous effort has been devoted to realizing such intriguing impurity-immunity in the surface waves of topological insulators as well as their electromagnetic analogue [25-28]. However, the nature of topological insulators only supports edge states instead of bulk states. To the best of our knowledge, the extraordinary property of electromagnetic impurity-immunity for bulk states has not been realized yet.

In this work, we demonstrate a principle to realize impurity-immunity for bulk electromagnetic waves, which leads to perfect transmission irrespective of embedded impurities of almost any material and shape. The property of electromagnetic impurity-immunity is induced by the introduction of a pair of parity-time $(P T)$-symmetric metasurfaces that sandwich a slab of ENZ medium, which 
is originally sensitive to impurities. In the past few years, significant efforts have been devoted to exploring the properties of the optical $P T$-symmetric systems with balanced gain and loss [29-53]. The optical analog of $P T$ symmetry [54] requires a refractive index profile of $n(\mathbf{r})=$ $n(-\mathbf{r})^{*}[29,30]$. The gain and loss in the optical materials allow the deviation from a conventional Hermitian system and introduce additional physics based on $P T$-phase transition and the associated exceptional points (EPs). Many intriguing phenomena have been discovered, such as power oscillations [31-33], coherent perfect absorption and lasing [34-38], unidirectional transparency [39-43], etc. More recently, the concept of $P T$ symmetry has been introduced in metasurfaces [55-57], which renders many exciting novel phenomena such as active negative refraction and imaging [46-49], unidirectional cloaking [50], electromagnetic teleportation [51], etc.

The electromagnetic impurity-immunity realized here comes from the EPs in the $P T$-symmetric system. In the phase diagram of $P T$-symmetric systems obtained by the variations of external parameters, EPs emerge as the unique phase transition points between the $P T$-symmetric and $P T$-broken phases. In the case of $P T$-symmetric metasurfaces, an interesting type of EP has been found at which the metasurfaces function as a pair of coherent perfect absorbers (CPAs) and lasers [46-49]. Here, by systematically investigating $P T$-symmetric metasurfaces, we observe a very different type of $\mathrm{EP}$ at which the metasurfaces function as a pair of unidirectional antireflection coatings (ARCs) for general dielectrics or metamaterials. By applying such $P T$-symmetric metasurfaces to ENZ media, we can compensate for the divergent impedance contrast between the ENZ media and free space. More importantly and interestingly, with such $P T$-symmetric metasurfaces, we find that the original "doping" effect of impurities in ENZ media [4] is significantly suppressed, and the perfect transmission becomes immune to two- and threedimensional impurities of almost any material and shape.

First, we consider a lossy metasurface (left) and a metasurface with gain (right) separated by a slab with relative permittivity $\varepsilon$ and relative permeability $\mu$, as illustrated in Fig. 1(a). The metasurfaces can be characterized by surface admittance $\pm\left|Y_{s}\right|$, or relative permittivity $\varepsilon_{m s}=1 \pm\left[\left(i\left|Y_{s}\right| / Y_{0}\right) / k_{0} d_{m s}\right]$, where $Y_{0}, k_{0}$, and $d_{m s}$ are the admittance of free space, the wave number in free space, and the thickness of the metasurface, respectively. The relative permeability of the metasurfaces is unity. When the surface admittances of the metasurfaces are opposite to each other, the optical $P T$-symmetric condition $n(-x)=$ $n(+x)^{*}$ is satisfied. In such a $P T$-symmetric system, the scattering matrix describing the relation between the incoming and outgoing waves can be written as

$$
S=\left(\begin{array}{cc}
t & r_{R} \\
r_{L} & t
\end{array}\right)
$$

where $r_{L(R)}$ is the reflection coefficient for left (right) incidence and $t$ is the transmission coefficient. Since the system is reciprocal, $t$ is identical for both left and right incidences. By considering $P T$ symmetry in Eq. (1), we can obtain the generalized energy-conservation relation [40]

$$
\sqrt{R_{L} R_{R}}=|T-1|,
$$

where $R_{L(R)} \equiv\left|r_{L(R)}\right|^{2}$ and $T \equiv|t|^{2}$ are, respectively, the reflectance and transmittance for left (right) incidence.

When $T<1$ ( or $T>1$ ), the eigenvalues of the scattering matrix are unimodular (or nonunimodular), indicating a $P T$-symmetric (or $P T$-broken) phase [36], in which case, the phase difference between $r_{L}$ and $r_{R}$ is zero (or $\pi$ ). The transition between the two phases happens at the EPs, at which $T=1$ and $R_{L} R_{R}=0$, indicating unity transmission and $R_{L}$ and/or $R_{R}$ is zero. As a result, at the EPs, $P T$-symmetric systems can exhibit the so-called unidirectional transparency [39].

By applying the requirement of $T=1$, the conditions of EPs for metasurfaces can be derived as [58]

$$
Y_{s}=\left(\cos \theta \pm \sqrt{\varepsilon / \mu-\sin ^{2} \theta / \mu^{2}}\right) Y_{0},
$$

for the case of transverse electric (TE, with electric fields in the $z$ direction) polarization, and

$$
Y_{s}=\left(\frac{1}{\cos \theta} \pm \frac{\varepsilon}{\sqrt{\varepsilon \mu-\sin ^{2} \theta}}\right) Y_{0},
$$

for the case of transverse magnetic (TM, with magnetic fields in the $z$ direction) polarization. Here, $\theta$ is the incidence angle in free space, which should be smaller than the critical angle of total reflection, i.e., $\arcsin \sqrt{\varepsilon \mu}$. The " \pm " sign indicates that there exist two solutions of EPs. When $Y_{s}>0$, the solution gives $R_{L}=0$ for incidence on the lossy metasurface, i.e., from the left-hand side. When $Y_{s}<0$, the solution gives $R_{R}=0$ for incidence on the gain metasurface, i.e., from the right-hand side. Interestingly, both solutions are independent of the thickness $d$ of the sandwiched slab. As we shall see later, the two solutions are complementary to each other but have very different physical nature.

In Fig. 1(b), we plot the phase diagram of the $P T$ symmetric system under an incident angle of $\theta=30^{\circ}$ for TE polarization. The slab sandwiched by the $P T$-symmetric metasurfaces is chosen as a dielectric medium with $\varepsilon=4$ and $\mu=1$. In Fig. 1(b), the white and gray regions represent the symmetric and broken phases, respectively, while EPs are between the $P T$-symmetric and $P T$-broken phases, i.e., $Y_{s}=1.07 Y_{0}$ and $Y_{s}=2.80 Y_{0}$, both independent of $d$. The two values are consistent with Eq. (3), with the choice of a minus or plus sign, respectively. In Figs. 1(c) and 1(d), we plot the reflectance $R_{L}$ and $R_{R}$, respectively, showing unidirectional transparency at the EPs.

We note that the additional white lines in Fig. 1(b) or the horizontal blue lines in Figs. 1(c) and 1(d) (periodic in $d$ ) in 

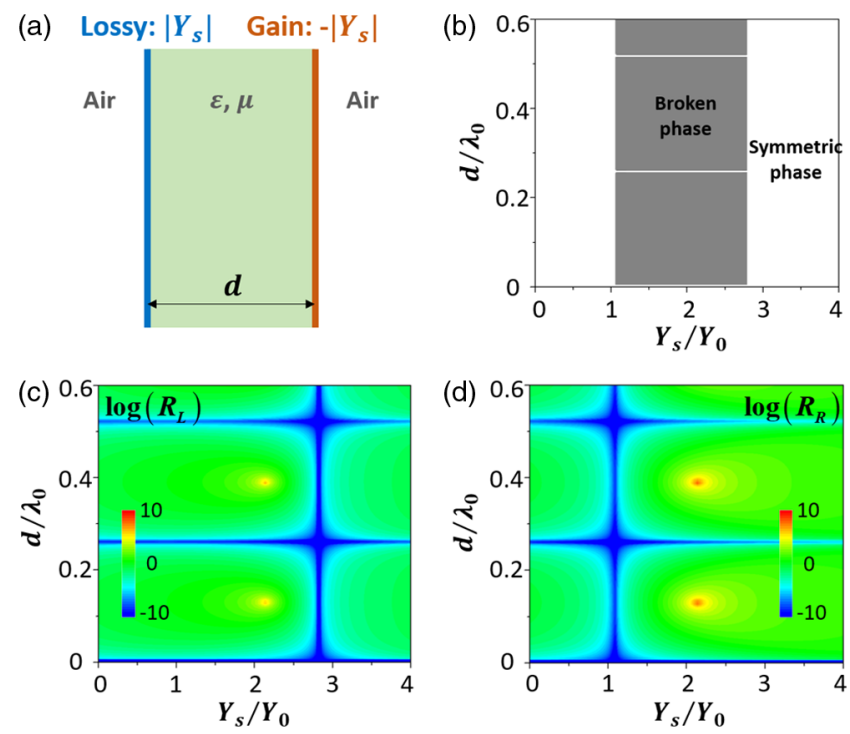

FIG. 1. (a) Illustration of the $P T$-symmetric system composed of a slab with relative permittivity $\varepsilon$ and relative permeability $\mu$ sandwiched by $P T$-symmetric metasurfaces with surface admittance $\pm\left|Y_{s}\right|$. (b) Phase diagram for incident angle $\theta=30^{\circ}$ under TE polarization. The white and gray regions denote the symmetric and broken phases, respectively. (c,d) Reflectance as functions of $d / \lambda_{0}$ and $Y_{s} / Y_{0}$ for (c) left and (d) right incidence on the $P T$-symmetric system. The red dots are related to the zeros and poles of the scattering matrix of the $P T$-symmetric system. In panels (b)-(d), the parameters of the slab are $\varepsilon=4$ and $\mu=1$.

the $P T$-broken phase are induced by Fabry-Pérot resonances of the slab and are therefore independent of $Y_{s}$. The condition can be easily derived as $d=m \pi /\left(k_{0} \sqrt{\varepsilon-\sin ^{2} \theta}\right)$, where $m$ is an integer. In this case, there is no reflection for either left or right incidence, as shown in Figs. 1(c) and 1(d).

Next, we demonstrate that the physical nature of the two EPs are very different. At the EP of $Y_{s}=1.07 Y_{0}, R_{R}=0$. In the upper panel of Fig. 2(b), we show the field distribution obtained by using COMSOL multiphysics software. We see that the angle of refraction is positive in this case. The energy flux flows from the right to the left inside the slab, which is the same as those in free space. Interestingly, there is no reflection on either surface of the dielectric slab. In this sense, the functionality of $P T$-symmetric metasurfaces is a pair of ARCs [59], denoted as an ARC-ARC pair, as illustrated in Fig. 2(a).

On the other hand, at the EP of $Y_{s}=2.80 Y_{0}, R_{L}=0$. In the field distribution shown in the upper panel of Fig. 2(d), we can see that the angle of refraction is negative in this case. The energy flux flows from the right to the left inside the slab, indicating that the lossy metasurface absorbs waves from both sides, while the metasurface with gain radiates toward both sides. In this sense, the functionality of $P T$-symmetric metasurfaces is a pair of coherent perfect absorption or lasing $[60,61]$, denoted as a CPA-laser pair, as illustrated in Fig. 2(c).

The wave behaviors at the EPs under TM polarization also exhibit the above two types, as demonstrated in the

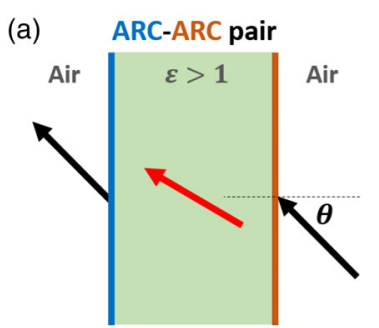

(b)

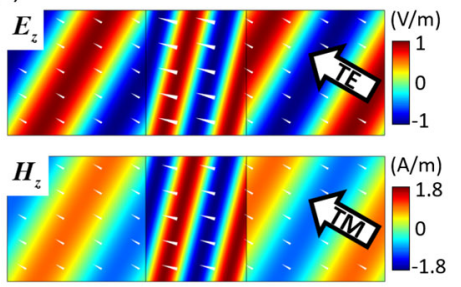

(c)

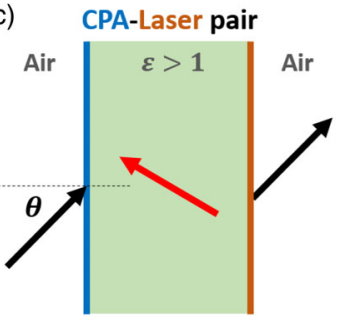

(d)

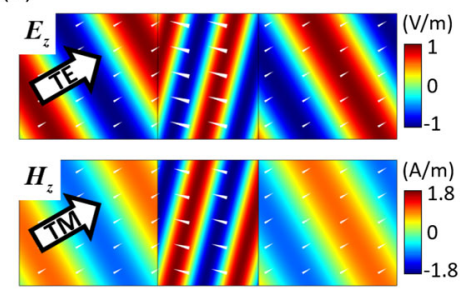

FIG. 2. (a) Illustration of the positive refraction in an ARCARC pair associated with the lower EP under the right incidence. (b) Simulated electric or magnetic fields: $E_{z}$ (TE polarization, $\left.Y_{s}=1.07 Y_{0}\right)$ or $H_{z}$ (TM polarization, $\left.Y_{s}=0.91 Y_{0}\right)$, and the time-averaged power flow (arrows) for the model in panel (a). (c) Illustration of the negative refraction in a CPA-laser pair associated with the upper EP under the left incidence. (d) Simulated electric or magnetic fields: $E_{z}$ (TE polarization, $\left.Y_{s}=2.80 Y_{0}\right)$ or $H_{z}$ (TM polarization, $Y_{s}=3.22 Y_{0}$ ), and the time-averaged power flow (arrows) for the model in panel (c). The incident angle in panels (b) and (d) is $\theta=30^{\circ}$.

lower panels of Figs. 2(b) and 2(d), respectively. We note that the EPs associated with the ARC-ARC pair have not been reported before because, previously, only free space was considered between the $P T$-symmetric metasurfaces [46-49], in which such EPs do not exist. Here, with both types of complementary EPs given by a unified formula, i.e., Eq. (3), the solutions of the EPs are now complete.

When the relative permittivity of the slab changes from $\varepsilon>1$ to $\varepsilon<1$, an interesting transition behavior appears for the EP associated with an ARC-ARC pair of metasurfaces. By assuming a nonmagnetic slab with $\mu=1$ and normal incidence with $\theta=0$, Eq. (3) is simplified to $Y_{s}=$ $(1-\sqrt{\varepsilon}) Y_{0}$ for the EP associated with an ARC-ARC pair. Apparently, $Y_{s}$ changes from $Y_{s}<0$ to $Y_{s}>0$ at the transition point of $\varepsilon=1$. This indicates a transition behavior from $R_{R}=0$ to $R_{L}=0$. When $\varepsilon<1$, to obtain unidirectional nonreflection, wave incidence is from the left-hand side, which is opposite from the case shown in Figs. 1 and 2 with $\varepsilon>1$. Such a transition behavior occurring at $\varepsilon=1$ is nonexistent for the EP associated with a CPA-laser pair because $Y_{s}=(1+\sqrt{\varepsilon}) Y_{0}$ is always positive.

Next, we consider the interesting case of an ENZ medium slab (with $\varepsilon=0.001$ and $\mu=1$ ) between the $P T$-symmetric metasurfaces, as shown in Fig. 3(a). In Fig. 3(b), we plot the phase diagram for normal incidence. Interestingly, the region of the $P T$-broken phase shrinks significantly. However, the EPs still exist, and both of them 

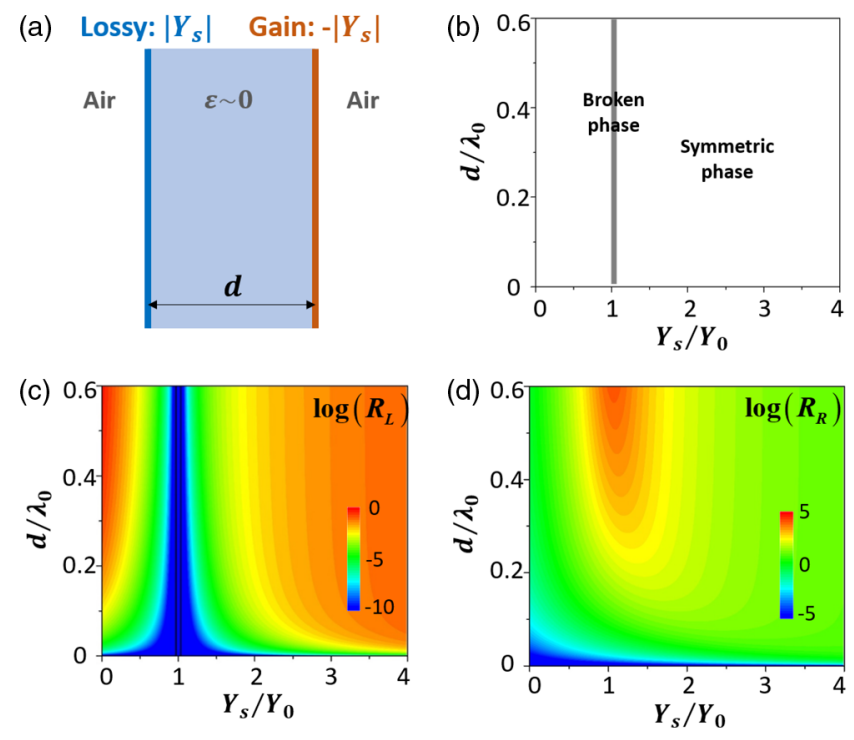

FIG. 3. (a) Illustration of the $P T$-symmetric system with $\varepsilon \approx 0$. (b) Phase diagram for normal incidence. The white and gray regions denote the symmetric phase and broken phase, respectively. [(c) and (d)] Reflectance as the functions of $d / \lambda_{0}$ and $Y_{s} / Y_{0}$ for (c) left and (d) right incidence on the $P T$-symmetric system.

converge to the same value of $Y_{s}=Y_{0}$. In other words, the two original solutions of EPs coalesce into one EP when $\varepsilon \rightarrow 0$ [58]. Figures 3(c) and 3(d) show, respectively, the reflectance $R_{L}$ and $R_{R}$. We notice that for both EPs, nonreflection is obtained for incidence from the left side because of the transition behavior for the EP associated with an ARC-ARC pair of metasurfaces.

In the following, we demonstrate that the properties of ENZ media undergo a fundamental change with the introduction of $P T$-symmetric metasurfaces. The well-known divergent impedance mismatch of ENZ media can be removed by the EPs. More importantly, the original sensitivity of impurities in ENZ media, which can tune the transmission behavior from total transmission to total reflection [4,12-20], can also be eliminated, leading to robust perfect transmission, irrespective of embedded impurities. In other words, the "doping" effect of ENZ media [4] is significantly suppressed, endowing the bulk system with the unprecedented property of impurity-immunity.

Through numerical simulations (using the COMSOL Multiphysics finite element software), we demonstrate the intriguing property of impurity-immunity for almost arbitrary impurities embedded in ENZ media, as illustrated in Fig. 4(a). For example, we consider a dielectric sphere of $\varepsilon_{d}=4$ and radius $R$ embedded in a slab of ENZ media (with $\varepsilon=10^{-3}+10^{-3} i$ ). In Fig. 4(b), we show the results obtained for $R_{L}, R_{R}$, and $T\left(=T_{L}=T_{R}\right)$ as a function of $R / \lambda_{0}$. Here, $T_{L}\left(T_{R}\right)$ denotes the transmittance for incidence from the left (right). When the $P T$-symmetric metasurfaces are applied, $R_{L} \rightarrow 0$ (blue dashed lines) and $T \approx 1$ (red dashed lines) irrespective of the radius of the sphere, $R$. However, we also see $R_{R} \neq 0$ [blue dotted

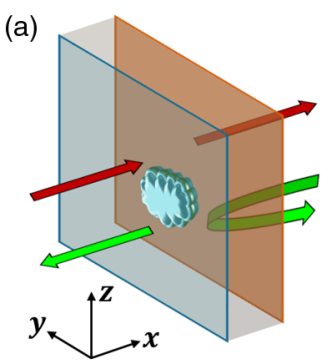

(c)

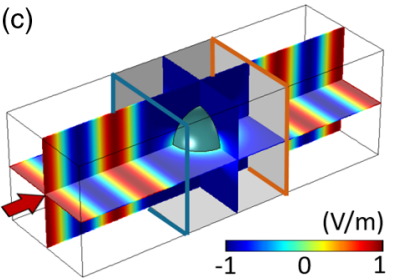

(e) Without metasurfaces

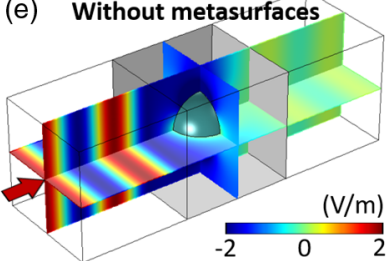

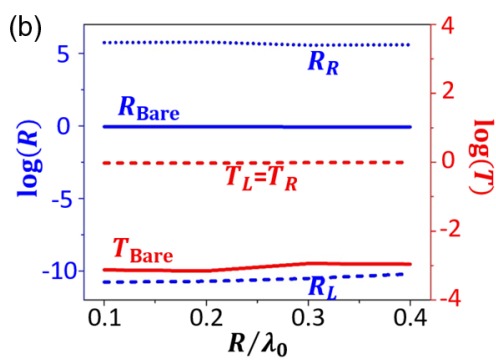

(d)

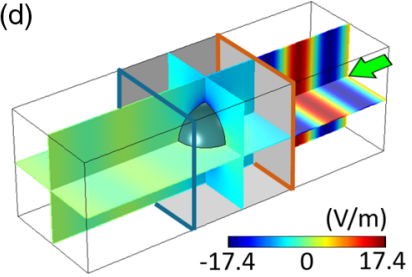

(f)

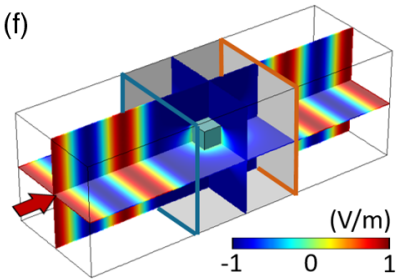

FIG. 4. (a) Illustration of the $P T$-symmetric metasurfaces separated by an ENZ medium slab embedded with an arbitrary three-dimensional object. (b) Reflectance and transmittance for an embedded dielectric sphere of $\varepsilon_{d}=4$ under the left (dashed lines) and right (dotted lines) incidence. (c)-(f) Distribution of $E_{z}$ in the ENZ medium slab for $(\mathrm{c}, \mathrm{f})$ the case with $P T$-symmetric metasurfaces for left incidence, (d) the case with $P T$-symmetric metasurfaces for right incidence, and (e) the case without $P T$-symmetric metasurfaces. The impurity is as follows: (c)-(e) A dielectric sphere of $\varepsilon_{d}=4$ and (f) a metallic cube of $\varepsilon_{d}=-2$. The relative permittivity of the ENZ medium is $\varepsilon=10^{-3}+10^{-3} i$.

lines in Fig. (b)], indicating that the perfect transmission is unidirectional. For comparison, when the $P T$-symmetric metasurfaces are removed, we observe that $R_{\mathrm{Bare}} \approx 1$ and $T_{\text {Bare }} \rightarrow 0$, i.e., almost total reflection and near-zero transmission due to the divergent impedance mismatch. We note that the surface admittance $Y_{s}=0.968 Y_{0}$ associated with the EP for the ARC-ARC pair is utilized for the demonstration of the impurity-immune effect here. Such an impurity-immunity effect can also be realized by using the EP for the CPA-laser pair, at which the surface admittance is $Y_{s}=1.032 Y_{0}$. The two EPs approach each other to the coalescence point of $Y_{s}=Y_{0}$ when $\varepsilon \rightarrow 0$.

The calculated field distributions are shown in Figs. 4(c)-4(e). In the simulations, the front and back boundaries (with the surface normal in the $y$ direction) are perfect magnetic conductors, and the upper and lower boundaries (with the surface normal in the $z$ direction) are perfect electric conductors so as to construct a waveguide supporting transverse electric and magnetic modes. Electromagnetic waves with electric fields polarized in 
the $z$ direction are incident from the left port boundary. The simulated electric fields $E_{z}$ in Figs. 4(c)-4(e) confirm the transmittance results in Fig. 4(b) for the ENZ medium slab of $d=1.5 \lambda_{0}$. In Fig. 4(f), we replace the dielectric sphere by a metallic cube of $\varepsilon_{d}=-2$ and still obtain $R_{L} \rightarrow 0$ and $T \approx 1$. This indicates that the perfect transmission is independent of the shape and size of the embedded impurity. Moreover, the effect is also independent of the thickness of the ENZ medium slab [58]. In this sense, $P T$ symmetric metasurfaces transform the ENZ media into a unidirectional impedance-matched media, which is immune to any three-dimensional impurities [21].

We have demonstrated the impurity-immunity phenomenon for isolated three-dimensional impurities like a sphere or a cube. Previously, in the case of a connected defect such as a cylindrical wire of infinite length, without the $P T$-symmetric metasurfaces currently considered, it has been demonstrated that these impurities in ENZ media would lead to interesting doping effects, which can be used to tune the transmission behavior from total transmission to total reflection $[4,12-20]$. We note that even the impedance-matched double-zero media (with both permittivity and permeability near zero) exhibit similar doping effects, resulting in impurity-dependent transmission properties $[13,14]$. Such doping effects can be understood as a consequence of the long-range connectivity of impurities $[21,22]$ in zero-index materials. On the contrary, here, by applying the $P T$-symmetric metasurfaces to ENZ media, the doping effect of continuous impurity can be suppressed, leading to impurity-immunity even for impurities with long-range connectivity, such as cylinders with infinite length. To demonstrate this important phenomenon, we place a dielectric cylinder of relative permittivity $\varepsilon_{d}=4$ with a random cross section inside the ENZ medium with the $P T$-symmetric metasurfaces, as shown in Fig. 5(a). The orientation is horizontal, so the system becomes a twodimensional one with TM polarization. Clearly, perfect transmission is observed. For comparison, in Fig. 5(b), we show that removal of the $P T$-symmetric metasurfaces leads to very low transmission. In Fig. 5(c), we show that even for an impedance-matched double-zero medium (without metasurfaces), the transmission is still very low because of the doping effect of the embedded cylinder. Moreover, in Fig. 5(d), we plot the transmittance $T$ as a function of $\varepsilon_{d}$ for the cases in Figs. 5(a) and 5(c). Clearly, without the $P T$-symmetric metasurfaces, $T$ varies dramatically as $\varepsilon_{d}$ increases, even for the impedance-matched double-zero medium, while for the ENZ medium with the $P T$-symmetric metasurfaces, we have $T \approx 1$, except for a very small region around a singular point at $\varepsilon_{d}=4.7$. When the ENZ medium approaches an ideal value, i.e., $\varepsilon \rightarrow 0$, the transmittance $T \rightarrow 1$, even near the singular point of $\varepsilon_{d}=4.7$.

Such extraordinary impurity-immunity effects for the infinite-sized impurities can actually be explained from the aspect of an effective medium by applying the doping
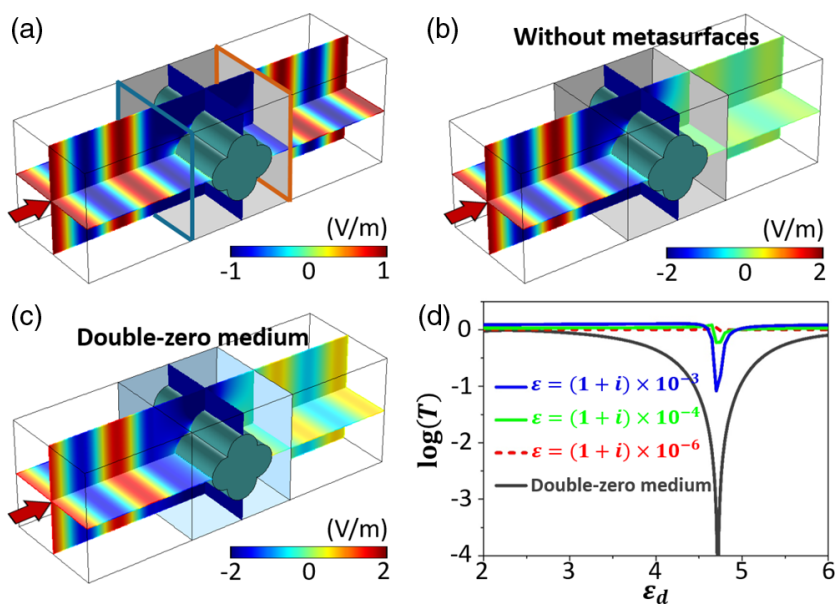

FIG. 5. (a)-(c) Distribution of $E_{z}$ for a dielectric cylinder of $\varepsilon_{d}=4$ with a random cross section embedded in (a) an ENZ medium $\left(\varepsilon=10^{-3}+10^{-3} i\right)$ with $P T$-symmetric metasurfaces, (b) an ENZ medium $\left(\varepsilon=10^{-3}+10^{-3} i\right)$ without metasurfaces, and (c) a double-zero medium $\left(\varepsilon=\mu=10^{-3}+10^{-3} i\right)$. (d) Transmittance as a function of the relative permittivity of the dielectric cylinder for the case of the double-zero medium without metasurfaces (gray solid lines) or the ENZ medium with the $P T$-symmetric metasurfaces (other lines).

theory [4]. In two dimensions, the ENZ medium with impurities embedded behaves as an effective medium with effective permittivity $\varepsilon_{\text {eff }} \approx 0$ and a tunable effective permeability $\mu_{\text {eff }}$. When $\mu_{\text {eff }} \rightarrow 0$, the ENZ medium turns into a double-zero medium, which enables total transmittance [4]. When $\mu_{\text {eff }}$ is a finite number, i.e., $\left|\mu_{\text {eff }}\right| \ll \infty$, Eq. (3) can be rewritten as $Y_{s}=\left(1 \pm \sqrt{\varepsilon_{\text {eff }} / \mu_{\text {eff }}}\right) Y_{0}$ [58]. From this equation, it can be seen that, as long as $\varepsilon_{\text {eff }} \rightarrow 0$, the EP solutions are $Y_{s} \approx Y_{0}$ for any finite-valued $\mu_{\mathrm{eff}}$. This indicates that the EP is almost independent of the impurities, leading to impurity-immune perfect transmission. In Fig. 5(d), we can see that, even near the singular point of $\varepsilon_{d}=4.7$, where $\left|\mu_{\text {eff }}\right| \rightarrow \infty[4,19]$, the transmission can be restored to near unity when the ENZ medium has $\varepsilon=10^{-6}+10^{-6} i$.

In the following, we apply field analysis to understand the physical mechanism of the impurity-immunity phenomenon. Without loss of generality, we consider the two-dimensional case under TM-polarized incidence (the same case as in Fig. 5) in which the magnetic field is in the out-of-plane direction, as schematically shown in Fig. 6. The origin of the doping effect is the tunable resonant magnetic field in the impurities. Because of the near-zero permittivity of the ENZ medium, the magnetic field $H_{\mathrm{ENZ}}$ is almost a constant in the ENZ medium, as shown in Fig. 6(a). Therefore, $H_{L} \approx H_{R} \approx H_{\mathrm{ENZ}}$, where $H_{L}$ and $H_{R}$ are the magnetic fields on the left and right boundaries of the ENZ medium, respectively. On the other hand, by using Faraday's law $\oint \mathbf{E} \cdot d \mathbf{l}=i \omega \int \mathbf{B} \cdot d \mathbf{S}, E_{L}$ and $E_{R}$, i.e., the tangential electric fields on the left and right boundaries of the ENZ 


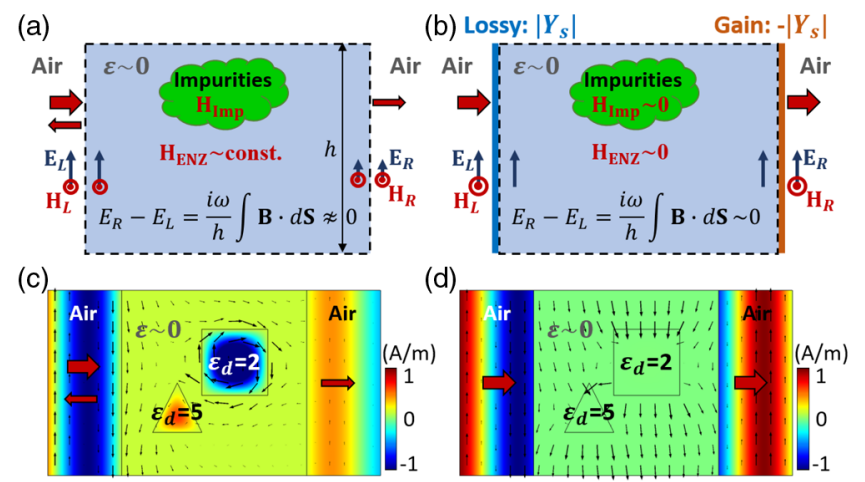

FIG. 6. (a,b) Schematic graphs of the electric- and magneticfield analysis for (a) the doping model without $P T$-symmetric metasurfaces and (b) the impurity-immunity model with $P T$ symmetric metasurfaces. The dashed lines denote the integral area for the calculation of magnetic flux. (c,d) The distributions of calculated electric fields (arrows) and magnetic fields (color) for the doping model and the impurity-immunity model with $P T$ symmetric metasurfaces, respectively. A triangular impurity of $\varepsilon_{d}=5$ and a square impurity of $\varepsilon_{d}=2$ exist in both cases.

medium, can be linked by the magnetic flux through the whole region bounded by the ENZ medium, including the ENZ medium itself and the embedded impurities, i.e.,

$$
E_{R}-E_{L}=\frac{i \omega}{h} \int \mathbf{B} \cdot d \mathbf{S},
$$

where $h$ is the height of the ENZ medium and $\mathbf{B}$ is the magnetic field. We note that when impurities are absent, the electric field decays linearly with the thickness of the ENZ medium slab (see, e.g., Ref. [62]). However, when impurities exist in the ENZ medium, there are resonant magnetic fields inside the impurities, i.e., $H_{\text {Imp }}$, as shown in Fig. 6(c). Note that $H_{\text {Imp }}$ will change the total magnetic flux $\int \mathbf{B} \cdot d \mathbf{S}$ of the whole area and the electric fields on the boundary of the ENZ medium, $E_{R}-E_{L}$, which will further change the transmittance through the whole system. Since $H_{\text {Imp }}$ strongly depends on the parameters of the impurities, the transmittance, as well as the effective medium of the system (see, e.g., Ref. [4]), can be tuned efficiently by the impurities, leading to the doping effect.

Interestingly, when the $P T$-symmetric metasurfaces are applied, the electric field still exists in the ENZ media, but the magnetic field is dramatically reduced to almost zero, as shown in Fig. 6(b). We note that across the ultrathin $P T$-symmetric metasurfaces with unity permeability, the tangential electric field is almost a constant, but the tangential magnetic field will experience a sharp change due to surface currents on the metasurfaces (see, e.g., Fig. 2 in the case of $H_{z}$ polarization). Specifically, at the EP where the metasurfaces satisfy Eq. (3), the left metasurface will work as a CPA or an ARC, so there is no reflection on the metasurface. In this case, considering the constant electric fields across the metasurface, the magnetic field inside the ENZ medium can be derived as $H_{\mathrm{ENZ}}=\sqrt{\varepsilon} H_{L}$. Here, $\varepsilon$ is the permittivity of the ENZ medium. When $\varepsilon \rightarrow 0$, the magnetic fields in the ENZ medium and impurities are also near zero, i.e., $H_{\mathrm{ENZ}} \sim 0$ and $H_{\mathrm{Imp}} \sim 0$. This is a consequence of the dramatic impedance difference between free space and the ENZ medium and the unidirectional transmission at the EP. Therefore, we obtain $E_{R}-E_{L}=$ $[(i \omega) /(h)] \int \mathbf{B} \cdot d \mathbf{S} \sim 0$, i.e., $E_{R} \approx E_{L}$. Since the tangential electric field across the metasurface is constant, the transmittance through the system will be restored to unity, and this property is independent of the embedded impurities. The above analysis explains the physical origin of the impurity-immunity phenomenon.

To verify the above field analysis, in Figs. 6(c) and 6(d), we plot the calculated electric- and magnetic-field distributions, for the doping model with a triangular impurity of $\varepsilon_{d}=5$ and a square impurity of $\varepsilon_{d}=2$, as well as the impurity-immunity model with $P T$-symmetric metasurfaces, respectively. Clearly, in the doping case without metasurfaces, $H_{\text {Imp }}$ is large inside the impurities, which can thus tune the total magnetic flux and lead to the doping effect. However, when the $P T$-symmetric metasurfaces are applied, $H_{\mathrm{ENZ}}$ and $H_{\mathrm{Imp}}$ are both reduced to almost zero, which guarantees perfect transmission and turns off the doping effect of the original impurities.

The origin of impurity-immunity lies in the near-zero magnetic flux in the region containing both ENZ medium and impurities, which occurs at $\varepsilon \rightarrow 0$. When $\varepsilon \rightarrow 0$, we also find that the region of the $P T$-broken phase diminishes, and the two EPs approach each other and coalesce into one EP. In this sense, the impurity-immunity phenomenon is associated with the coalescence of EPs. The coalescence and high order of EPs [63-66] are interesting phenomena with many important applications such as enhanced sensitivity. Here, we have demonstrated another interesting consequence of the coalescence of EP in the transmission behavior of a $P T$-symmetric system with ENZ medium. We have plotted and discussed the coalescence process of the EPs in Ref. [58].

The electric fields throughout the ENZ media serve as a bridge linking the incident and transmitted waves. Such electric fields are also nonvariant across both metasurfaces because of the ultrathin thickness and $\mu=1$. More interestingly, the electric fields can link unparalleled $P T$-symmetric metasurfaces, enabling more general-shaped configurations beyond the above planar configurations, as we have shown in Ref. [58].

One may notice that in our simulations, the impurityimmunity phenomenon is demonstrated in the existence of the material loss of the ENZ media. A detailed analysis of the effects of loss in both the ENZ media and the impurities is presented in Ref. [58].

The above results have demonstrated the impurityimmunity phenomenon under normal incidence. The condition of normal incidence can usually be realized by using 
wide-aperture coherent incident beams or waveguide structures. In practice, the permittivity of the ENZ medium has a small but finite value. In such cases, the impurity-immunity phenomenon can be extended to slightly oblique incidence [58]. From Eq. (3), it can be seen that weak nonlocality is required in the ideal parameters of metasurfaces under oblique incidence. The concept and design methods of such nonlocal metasurfaces have previously been developed in Refs. [48,49].

Practical implementation of the $P T$-symmetric metasurfaces can be realized in the microwave and optical frequency regimes. The lossy metasurfaces can be simply achieved by conductive films [59-61]. In THz and optical designs, the conductive films can be composed of conductive materials like chromium [67], gold [68], graphene [69], indium tin oxide [70], etc. On the other hand, the metasurfaces with gain can be achieved by employing gain structures in metasurface or metamaterial designs. For instance, a grating of wide metallic strips periodically loaded with lumped elements has been proposed to design the metasurfaces with gain for microwave cloaking [50]. In addition, subwavelength structures with a large amount of gain can be realized by using multilayered structures [48], microwave and optical metastructures with gain [71-75], etc. In particular, the effective gain media with pure permittivity or permeability have also been demonstrated in core-shell composites [76]. These works manifest the possibility of realizing the metasurfaces with the required effective gain parameters.

Finally, we have also investigated the stability of our $P T$-symmetric system by plotting the poles of the scattering matrix on the complex frequency plane, as reported in Ref. [58]. By applying appropriate Lorentz and antiLorentz dispersion models to the metasurfaces and a Drude dispersion model to the ENZ medium, we find that all poles can be confined to the lower half-plane, indicating that our system can be unconditionally stable for arbitrary temporal excitations [48-50]. Moreover, the position change of the poles caused by the embedded impurity is almost negligible, confirming the stability and the feasibility of the proposed $P T$-symmetric system, as well as the robustness of the impurity-immunity functionality.

To conclude, we have analytically given the complete set of EPs for $P T$-symmetric metasurfaces sandwiching a slab of dielectric-metamaterial slab. A new type of EP at which the metasurfaces function as ARCs is revealed. By applying such metasurfaces to a slab of ENZ medium, the original two types of EPs coalesce into one EP. Interestingly, at this critical point of $\varepsilon \rightarrow 0$, not only can the significant issue of a huge impedance mismatch with free space be solved, but the doping effect of ENZ medium can also be significantly suppressed, leading to the unprecedented property of electromagnetic impurity-immunity for bulk electromagnetic waves. This physical mechanism of the impurity-immunity phenomenon lies in the near-zero magnetic flux in the region bounded by the ENZ medium; thus, the principle applies for impurities of almost arbitrary shapes and materials. Our work demonstrates that, with proper design, $P T$-symmetry with EPs can be utilized to realize the rare and valuable phenomenon of impurityimmunity for bulk states.

\section{ACKNOWLEDGMENTS}

We sincerely thank Dr. Kun Ding for the helpful discussions. This work is supported by National Natural Science Foundation of China (Grants No. 61671314, No. 11574226, and No. 11704271), Natural Science Foundation of Jiangsu Province (Grant No. BK20170326), Natural Science Foundation for Colleges and Universities in Jiangsu Province of China (Grant No. 17KJB140019), Jiangsu Planned Projects for Postdoctoral Research Funds (1701181B), and a Project Funded by the Priority Academic Program Development of Jiangsu Higher Education Institutions (PAPD). J. L. acknowledges funding from the William Mong Institute of Nano Science and Technology of the Hong Kong University of Science and Technology.

[1] J. C. Maxwell Garnett, Colours in Metal Glasses and in Metallic Films, Phil. Trans. R. Soc. A 203, 385 (1904).

[2] D. A. G. Bruggeman, Calculation of Various Physics Constants in Heterogenous Substances I. Dielectricity Constants and Conductivity of Mixed Bodies from Isotropic Substances, Ann. Phys. (Berlin) 416, 636 (1935).

[3] K. Dolgaleva and R. W. Boyd, Local-Field Effects in Nanostructured Photonic Materials, Adv. Opt. Photonics 4, 1 (2012).

[4] I. Liberal, A. M. Mahmoud, Y. Li, B. Edwards, and N. Engheta, Photonic Doping of Epsilon-Near-Zero Media, Science 355, 1058 (2017).

[5] I. Liberal and N. Engheta, Near-Zero Refractive Index Photonics, Nat. Photonics 11, 149 (2017).

[6] N. Engheta, Pursuing Near-Zero Response, Science 340, 286 (2013).

[7] S. Enoch, G. Tayeb, P. Sabouroux, N. Guérin, and P. Vincent, A Metamaterial for Directive Emission, Phys. Rev. Lett. 89, 213902 (2002).

[8] R. Ziolkowski, Propagation in and Scattering from a Matched Metamaterial Having a Zero Index of Refraction, Phys. Rev. E 70, 046608 (2004).

[9] M. Silveirinha and N. Engheta, Tunneling of Electromagnetic Energy through Subwavelength Channels and Bends Using $\epsilon$-Near-Zero Materials, Phys. Rev. Lett. 97, 157403 (2006).

[10] J. Luo, W. Lu, Z. Hang, H. Chen, B. Hou, Y. Lai, and C. T. Chan, Arbitrary Control of Electromagnetic Flux in Inhomogeneous Anisotropic Media with Near-Zero Index, Phys. Rev. Lett. 112, 073903 (2014).

[11] M. Zhou, L. Shi, J. Zi, and Z. Yu, Extraordinarily Large Optical Cross Section for Localized Single Nanoresonator, Phys. Rev. Lett. 115, 023903 (2015). 
[12] M. Silveirinha and N. Engheta, Design of Matched ZeroIndex Metamaterials Using Nonmagnetic Inclusions in Epsilon-Near-Zero Media, Phys. Rev. B 75, 075119 (2007).

[13] J. Hao, W. Yan, and M. Qiu, Super-Reflection and Cloaking Based on Zero Index Metamaterial, Appl. Phys. Lett. 96, 101109 (2010).

[14] V. C. Nguyen, L. Chen, and K. Halterman, Total Transmission and Total Reflection by Zero Index Metamaterials with Defects, Phys. Rev. Lett. 105, 233908 (2010).

[15] Y. Xu and H. Chen, Total Reflection and Transmission by Epsilon-Near-Zero Metamaterials with Defects, Appl. Phys. Lett. 98, 113501 (2011).

[16] X. Huang, Y. Lai, Z. H. Hang, H. Zheng, and C. T. Chan, Dirac Cones Induced by Accidental Degeneracy in Photonic Crystals and Zero-Refractive-Index Materials, Nat. Mater. 10, 582 (2011).

[17] J. Luo, P. Xu, L. Gao, Y. Lai, and H. Chen, Manipulate the Transmissions Using Index-Near-Zero or Epsilon-NearZero Metamaterials with Coated Defects, Plasmonics 7, 353 (2012).

[18] Y. Wu and J. Li, Total Reflection and Cloaking by Zero Index Metamaterials Loaded with Rectangular Dielectric Defects, Appl. Phys. Lett. 102, 183105 (2013).

[19] T. Wang, J. Luo, L. Gao, P. Xu, and Y. Lai, Equivalent Perfect Magnetic Conductor Based on Epsilon-Near-Zero Media, Appl. Phys. Lett. 104, 211904 (2014).

[20] A. M. Mahmoud and N. Engheta, Wave-Matter Interactions in Epsilon-and-Mu-Near-Zero Structures, Nat. Commun. 5, 5638 (2014).

[21] J. Luo, Z.H. Hang, C. T. Chan, and Y. Lai, Unusual Percolation Threshold of Electromagnetic Waves in Double-Zero Medium Embedded with Random Inclusions, Laser Photonics Rev. 9, 523 (2015).

[22] A. S. Shalin, P. Ginzburg, A. A. Orlov, I. Iorsh, P. A. Belov, Y.S. Kivshar, and A. V. Zayats, Scattering Suppression from Arbitrary Objects in Spatially Dispersive Layered Metamaterials, Phys. Rev. B 91, 125426 (2015).

[23] M. I. Molina and G. P. Tsironis, Nonlinear Impurities in a Linear Chain, Phys. Rev. B 47, 15330(R) (1993).

[24] M. I. Molina and G. P. Tsironis, Absence of Localization in a Nonlinear Random Binary Alloy, Phys. Rev. Lett. 73, 464 (1994).

[25] R. E. Prange and S. M. Girvin, The Quantum Hall Effect (Springer-Verlag, Berlin, 1990).

[26] F. Haldane and S. Raghu, Possible Realization of Directional Optical Waveguides in Photonic Crystals with Broken TimeReversal Symmetry, Phys. Rev. Lett. 100, 013904 (2008).

[27] Z. Wang, Y. Chong, J. Joannopoulos, and M. Soljačić, Reflection-Free One-Way Edge Modes in a Gyromagnetic Photonic Crystal, Phys. Rev. Lett. 100, 013905 (2008).

[28] Z. Wang, Y. Chong, J. D. Joannopoulos, and M. Soljacic, Observation of Unidirectional Backscattering-Immune Topological Electromagnetic States, Nature (London) 461, 772 (2009).

[29] A. Ruschhaupt, F. Delgado, and J. G. Muga, Physical Realization of $\mathcal{P T}$-Symmetric Potential Scattering in a Planar Slab Waveguide, J. Phys. A 38, L171 (2005).

[30] R. El-Ganainy, K. G. Makris, D. N. Christodoulides, and Z. H. Musslimani, Theory of Coupled Optical PT-Symmetric Structures, Opt. Lett. 32, 2632 (2007).
[31] K. G. Makris, R. El-Ganainy, D. N. Christodoulides, and Z. H. Musslimani, Beam Dynamics in PT Symmetric Optical Lattices, Phys. Rev. Lett. 100, 103904 (2008).

[32] C.E. Rüter, K. G. Makris, R. El-Ganainy, D. N. Christodoulides, M. Segev, and D. Kip, Observation of Parity-Time Symmetry in Optics, Nat. Phys. 6, 192 (2010).

[33] M. C. Zheng, D. N. Christodoulides, R. Fleischmann, and T. Kottos, PT Optical Lattices and Universality in Beam Dynamics, Phys. Rev. A 82, 010103(R) (2010).

[34] S. Longhi, PT-Symmetric Laser Absorber, Phys. Rev. A 82, 031801(R) (2010).

[35] H. Schomerus, Quantum Noise and Self-Sustained Radiation of PT-Symmetric Systems, Phys. Rev. Lett. 104, 233601 (2010).

[36] Y. D. Chong, L. Ge, and A. D. Stone, PT-Symmetry Breaking and Laser-Absorber Modes in Optical Scattering Systems, Phys. Rev. Lett. 106, 093902 (2011).

[37] Y. Sun, W. Tan, H. Li, J. Li, and H. Chen, Experimental Demonstration of a Coherent Perfect Absorber with PT Phase Transition, Phys. Rev. Lett. 112, 143903 (2014).

[38] Z. J. Wong, Y. Xu, J. Kim, K. O'Brien, Y. Wang, L. Feng, and X. Zhang, Lasing and Anti-lasing in a Single Cavity, Nat. Photonics 10, 796 (2016).

[39] Z. Lin, H. Ramezani, T. Eichelkraut, T. Kottos, H. Cao, and D. N. Christodoulides, Unidirectional Invisibility Induced by PT-Symmetric Periodic Structures, Phys. Rev. Lett. 106, 213901 (2011).

[40] L. Ge, Y. D. Chong, and A. D. Stone, Conservation Relations and Anisotropic Transmission Resonances in OneDimensional PT-Symmetric Photonic Heterostructures, Phys. Rev. A 85, 023802 (2012).

[41] A. Regensburger, C. Bersch, M. A. Miri, G. Onishchukov, D. N. Christodoulides, and U. Peschel, Parity-Time Synthetic Photonic Lattices, Nature (London) 488, 167 (2012).

[42] L. Feng, Y. L. Xu, W. S. Fegadolli, M. H. Lu, J. E. Oliveira, V. R. Almeida, Y.F. Chen, and A. Scherer, Experimental Demonstration of a Unidirectional Reflectionless ParityTime Metamaterial at Optical Frequencies, Nat. Mater. 12, 108 (2013).

[43] H. Ramezani, H. Li, Y. Wang, and X. Zhang, Unidirectional Spectral Singularities, Phys. Rev. Lett. 113, 263905 (2014).

[44] X. Zhu, L. Feng, P. Zhang, X. Yin, and X. Zhang, One-Way Invisible Cloak Using Parity-Time Symmetric Transformation Optics, Opt. Lett. 38, 2821 (2013).

[45] J. Gear, F. Liu, S. T. Chu, S. Rotter, and J. Li, Parity-Time Symmetry from Stacking Purely Dielectric and Magnetic Slabs, Phys. Rev. A 91, 033825 (2015).

[46] R. Fleury, D. L. Sounas, and A. Alù, Negative Refraction and Planar Focusing Based on Parity-Time Symmetric Metasurfaces, Phys. Rev. Lett. 113, 023903 (2014).

[47] C. A. Valagiannopoulos, F. Monticone, and A. Alù, PT-Symmetric Planar Devices for Field Transformation and Imaging, J. Opt. 18, 044028 (2016).

[48] F. Monticone, C. A. Valagiannopoulos, and A. Alù, ParityTime Symmetric Nonlocal Metasurfaces: All-Angle Negative Refraction and Volumetric Imaging, Phys. Rev. X 6, 041018 (2016).

[49] S. Savoia, C. A. Valagiannopoulos, F. Monticone, G. Castaldi, and V. Galdi, Magnified Imaging Based on 
Non-Hermitian Nonlocal Cylindrical Metasurfaces, Phys. Rev. B 95, 115114 (2017).

[50] D. L. Sounas, R. Fleury, and A. Alù, Unidirectional Cloaking Based on Metasurfaces with Balanced Loss and Gain, Phys. Rev. Applied 4, 014005 (2015).

[51] Y. Ra'Di, D. L. Sounas, A. Alù, and S. A. Tretyakov, ParityTime-Symmetric Teleportation, Phys. Rev. B 93, 235427 (2016).

[52] S. Xiao, J. Gear, S. Rotter, and J. Li, Effective PT-Symmetric Metasurfaces for Subwavelength Amplified Sensing, New J. Phys. 18, 085004 (2016).

[53] L. Feng, R. El Ganainy, and L. Ge, Non-Hermitian Photonics Based on Parity-Time Symmetry, Nat. Photonics 11, 752 (2017).

[54] C. M. Bender and S. Boettcher, Real Spectra in NonHermitian Hamiltonians Having PT Symmetry, Phys. Rev. Lett. 80, 5243 (1998).

[55] N. Yu, P. Genevet, M. A. Kats, F. Aieta, J. Tetienne, F. Capasso, and Z. Gaburro, Light Propagation with Phase Discontinuities: Generalized Laws of Reflection and Refraction, Science 334, 333 (2011).

[56] X. Ni, N. K. Emani, A. V. Kildishev, A. Boltasseva, and V. M. Shalaev, Broadband Light Bending with Plasmonic Nanoantennas, Science 335, 427 (2012).

[57] S. Sun, Q. He, S. Xiao, Q. Xu, X. Li, and L. Zhou, GradientIndex Meta-surfaces as a Bridge Linking Propagating Waves and Surface Waves, Nat. Mater. 11, 426 (2012).

[58] See Supplemental Material at http://link.aps.org/ supplemental/10.1103/PhysRevX.8.031035 for details of derivation of formula, analysis of TM polarization, opposite incidence, effects of the thickness of ENZ media and metasurfaces, ENZ and double-zero media with impurities, influences of material loss, impurity-immunity under oblique incidence and in general geometrical configurations, stability of the system, coalescence of exceptional points.

[59] J. Luo, S. Li, B. Hou, and Y. Lai, Loss/Gain-Induced Ultrathin Antireflection Coatings, Sci. Rep. 6, 28681 (2016).

[60] J. Luo, S. Li, B. Hou, and Y. Lai, Unified Theory for Perfect Absorption in Ultrathin Absorptive Films with Constant Tangential Electric or Magnetic Fields, Phys. Rev. B 90, 165128 (2014).

[61] S. Li, J. Luo, S. Anwar, S. Li, W. Lu, Z. H. Hang, Y. Lai, B. Hou, M. Shen, and C. Wang, Broadband Perfect Absorption of Ultrathin Conductive Films with Coherent Illumination: Superabsorption of Microwave Radiation, Phys. Rev. B 91, 220301(R) (2015).

[62] A. Alù, M. Silveirinha, A. Salandrino, and N. Engheta, Epsilon-Near-Zero Metamaterials and Electromagnetic
Sources: Tailoring the Radiation Phase Pattern, Phys. Rev. B 75, 155410 (2007).

[63] J. Wiersig, Enhancing the Sensitivity of Frequency and Energy Splitting Detection by Using Exceptional Points: Application to Microcavity Sensors for Single-Particle Detection, Phys. Rev. Lett. 112, 203901 (2014).

[64] K. Ding, G. Ma, M. Xiao, Z. Q. Zhang, and C. T. Chan, Emergence, Coalescence, and Topological Properties of Multiple Exceptional Points and Their Experimental Realization, Phys. Rev. X 6, 021007 (2016).

[65] K. Ding, Z. Q. Zhang, and C. T. Chan, Coalescence of Exceptional Points and Phase Diagrams for OneDimensional PT-Symmetric Photonic Crystals, Phys. Rev. B 92, 235310 (2015).

[66] H. Hodaei, A. U. Hassan, S. Wittek, H. Garcia-Gracia, R. El-Ganainy, D. N. Christodoulides, and M. Khajavikhan, Enhanced Sensitivity at Higher-Order Exceptional Points, Nature (London) 548, 187 (2017).

[67] J. Kroll, J. Darmo, and K. Unterrainer, Metallic WaveImpedance Matching Layers for Broadband Terahertz. Optical Systems, Opt. Express 15, 6552 (2007).

[68] A. Thoman, A. Kern, H. Helm, and M. Walther, Nanostructured Gold Films as Broadband Terahertz Antireflection Coatings, Phys. Rev. B 77, 195405 (2008).

[69] S. Thongrattanasiri, F. H. L. Koppens, and F. J. García De Abajo, Complete Optical Absorption in Periodically Patterned Graphene, Phys. Rev. Lett. 108, 047401 (2012).

[70] L. Ding, X. Wang, N. S. S. Ang, C. Lu, V. Suresh, S. Chua, and J. Teng, Ultrathin Film Broadband Terahertz Antireflection Coating Based on Impedance Matching Method, IEEE J. Sel. Top. Quantum Electron. 23, 8500508 (2017).

[71] D. Ye, K. Chang, L. Ran, and H. Xin, Microwave Gain Medium with Negative Refractive Index, Nat. Commun. 5, 5841 (2014).

[72] S. Assawaworrarit, X. Yu, and S. Fan, Robust Wireless Power Transfer Using a Nonlinear Parity-Time-Symmetric Circuit, Nature (London) 546, 387 (2017).

[73] S. Xiao, V. P. Drachev, A. V. Kildishev, X. Ni, U. K. Chettiar, H. K. Yuan, and V. M. Shalaev, Loss-Free and Active Optical Negative-Index Metamaterials, Nature (London) 466, 735 (2010).

[74] O. Hess and K. L. Tsakmakidis, Metamaterials with Quantum Gain, Science 339, 654 (2013).

[75] P. Miao, Z. Zhang, J. Sun, W. Walasik, S. Longhi, N. M. Litchinitser, and L. Feng, Orbital Angular Momentum Microlaser, Science 353, 464 (2016).

[76] P. Bai, K. Ding, G. Wang, J. Luo, Z. Zhang, C. T. Chan, Y. Wu, and Y. Lai, Simultaneous Realization of a Coherent Perfect Absorber and Laser by Zero-Index Media with Both Gain and Loss, Phys. Rev. A 94, 063841 (2016). 